\title{
EYE MOVEMENT DESENSITIZATION AND REPROCESSING AS A PSYCHOTHERAPEUTIC APPROACH
}

\author{
Desislava Ivanova \\ South-West Univerisity "NeofitRilski", Bulgaria \\ E-mail: desi_hvr@yahoo.com
}

\begin{abstract}
Eye movement desensitization and reprocessing (EMDR) is empirically validated psychotherapeutic approach, which uses Adaptive Information Processing (AIP) model. EMDR can be applied when working with the consequences of psychological trauma and a wide range of other negative life experiences, excluding organic deficits, intoxication, and injury. EMDR therapy is conceptualized in eight phases, consisting of standardized protocols and procedures, which make easier the complete assessment of the clinical picture, patient preparation and the treatment of: past events, which place the foundations of pathology, current adverse situations and future challenges. The main focus of the therapy is the system that processes information in the brain. All dysfunctional characteristic traits, behaviors, beliefs, affects, and physical sensations are viewed from the perspective of (AIP) model as manifestations of unprocessed memories, encoded during a traumatic experience.
\end{abstract}

Keywords: EMDR therapy, psychotherapy approach, AIP model, reprocessing information.

\section{Introduction}

Eye movement desensitization and reprocessing (EMDR) is an empirically validated integrative psychotherapeutic approach, which is applied for intervention of a psychological trauma and other negative life experiences. The method is developed by Francine Shapiro and it is based on the Adaptive Information Processing (AIP) model, which is the basis to the understanding of clinical phenomenology and it focuses on the client's resources (Rogers \& Silver, 2002). The AIP method suggests that the human brain can usually process stressful information, in such a way that provides an adaptive response, creating functional memory networks. Only if this congenital system for information processing is affected, the memory will be stored "raw," "unprocessed" and in an "inappropriate" form, in a way that generates discomfort and clinical symptoms (van der Vleugel, van den Berg, \& Staring, 2012). This way, if the memory networks contain unprocessed materials, the present perceptions can be determined by earlier dysfunctional emotions, thoughts, beliefs and feelings, connected to the past event. This suggests the inability to connect with other memory networks, that contain adaptive information. The AIP model views the dysfunctionally stored memory networks as the hidden source of the pathology as well as the mental health (Solomon \& Shapiro, 2008). For example, a traumatic experience from the childhood can be coded as a 
survival mechanism that contains feelings of fear, which are inappropriate for an adult. However, these past events keep their intensity, because they have not been integrated in a suitable way in the adaptive networks (Rogers \& Silver, 2002). One key principle of the AIP model is that these dysfunctionally stored and not completely processed memories form the basis of psychopathology (Valiente-Gómez, Moreno-Alcázar, Treen, Cedrón, Colom, Pérez, \& Amann, 2017). The activation of these memories, even years after the event, can bring to a spectrum of symptoms that form the actual problems of the client. During the work process, by following the EMDR protocols and procedures, the dysfunctionally stored memories are processed by transforming the unprocessed memory components (visual pictures, thoughts, sounds, emotions, kinesthetic feelings, beliefs) in adaptive solutions. The AIP model does not accept the attitudes, emotions and sensations as simple reactions to past events. They are viewed as manifestations of physiologically stored perceptions, stored in the memory, and the reactions to them (Knalfa \&Touzet, 2017). This outlook on the present symptoms as a result of the activation of memories, which have not been adequately processed and stored, is an inseparable part of the therapeutic EMDR conception.

\section{Eye Movement Desensitization and Reprocessing (EMDR) Protocol in Eight Phases}

EMDR is a psychotherapeutic approach, which begins with the first contact with the client and unfolds into eight phases. The phases from three to eight are repeated in most of the sessions (Shapiro \& Maxfield, 2002). The understanding of the client and the clinical picture, the conceptualization of the case and therapeutic planning are based on the AIP model, according to which the present difficulties derive from inadequately processed past experiences that are stored in the nervous system in an inadequate way (De Jongh, Ten Broeke, \& Meije, 2010). Before turning attention to the traumatic memories, the therapist uses EMDR, to help the client develop his abilities, in order to be able to tolerate all negative effects that can be caused by activating the memory network. Although, the memory network contains information from the past, it serves in the present and future. All memories, which are processed, turn into resources, and the unprocessed ones into a pathology (Engelhard, van Uijen, \& ven den Hout, 2010). The understanding of AIP is the basis of the three-pronged protocol in EMDR, which accesses and processes with the past distressing events, presents triggers and future similar experiences. In order to provide complete processing of all connected to memory networks, the therapy usually begins with the earliest incidents (McCullough, 2002). In addition to guiding through and resolving traumatic memories, EMDR is used to handle current situations that cause emotional disturbances, by processing the triggers, in order to stop the activation of symptomatic reactions. It is also applied to help the client develop the specific skills and behavioral models needed for a healthy and functional life. According to a number of researches (Jeffries \& Davis, 2012; Shapiro, 2014) the bilateral stimulation (eye movements), as a component of EMDR, eases the information processing by decreasing the brightness of memory images and the influence connected with them. This effect can improve processing through desensitization by reducing the stress and the connected with this avoidance. It is supposed that because the image becomes less intrusive, the clients become more able to gain access to more adaptive information and create new connections in the memory network (Maxfield, Melnyk, \& Gordon Hayman, 2008). During the first phase the therapist gathers the complete history of the client by determining if EMDR is suitable for the case. Identifying the target events according to the positive and negative in the client's life, assessing his readiness for EMDR he develops a therapeutic plan. In addition to the standard procedures for assessment, the therapist identifies suitable goals for the therapy. The goals can include the development of resources for emotion management, disturbing memories and connected to them past incidents, presenting provocative factors that cause distress, as well as installing of patterns for future reference. Assessing the client's characteristics (stability, integrative capacity, tolerance to affect, history of attachment, readiness to change) during this phase of history taking is critically important for the successful course of the therapy.

The second phase of preparation or stabilization is directed towards establishing the 
therapeutic relationship, formulating reasonable expectations and educating the client about his symptoms. The focus is on improvement and development of personal resources, for example Vol. 12, No. 1, 2018 safety, teaching self-control techniques, before working on traumatic memories. At this point it is suggested to ease the access to positive emotions by creating a "safe place". These techniques for self-comforting are an important element of the therapy and are used to "close" incomplete sessions, and also to maintain the stability of the clients between and during séances. Clients with a history of childhood trauma often have a deficit in regulating the affect and impulse control and can require a significant preparation. For such clients, the interventions from the second phase can be prolonged significantly and to require work to improve the resources, combining relaxation and EMDR. This is applicable for some clients with anxiety disorders that manifest avoidance behavior, before beginning serious attempts to process the traumatic memories (Shapiro \& Maxfield, 2002).

In the third phase, access to the targeted memory is achieved and the processing of traumatic incidents begins, with structured clinically targeted assessment of the sensory, cognitive and affective components of the target memory (Patihis, Cruz, \& McNally, in press). The client describes the connected visual image, which is most vivid and convincing. After identifying the present irrational negative belief, that is provoked by this image (for example "I am a disappointment" or "I am weak"), the client expresses the wanted positive cognition (for example "I am important" or "I can do it"). Assessing how true is that positive belief, when thinking about the traumatic incident using the validity of the cognition (VOC) scale, where 1 is "feels completely false," and 7 is "feels completely true." By repeating the positive belief about him forms a realistic goal of the therapy. The client identifies the emotions associated with the targeted memory, by repeating the negative cognition. Besides that, it can speed up the processing of information, by creating preliminary associative connections between the target memory and the information correcting the emotion. After that the client combines the visual image with a negative belief - this activates the memory network and often causes a severe affect. The client identifies the emotions associated with the target incident and assesses his level of disturbance, tension, discomfort with Subjective Unit of Disturbance (SUD) scale, where 0 is "completely relaxed" and 10 is "the highest disturbance imaginable." The emotion intensity rating allows the client to express feelings, which may have never been expressed before, this way makes the preliminary processing of the information easier. Besides this the direction of the response allows the client and the therapist to recognize changes in the type of emotion, experienced during the session. Subsequently, the client identifies and discovers the physical sensations that are caused by the traumatic image. The answer of the question: "Where in the body do you feel it?" enables to specify the sensory symptoms (e.g. nausea, sweating, palpitations) and to differentiate them from cognitive interpretations (for example: I'm powerless). To summarize, in phase three the image, the negative belief, which is present at the moment, the desired positive belief, the present emotion, the physical sensation are revealed and the levels are measured according to the basic scales validity of cognition (VOC) scale and Subjective Unit of Disturbance (SUD) scale.

In phase four, desensitization is achieved. The chains of associations are being followed, in the order in which they appear with regard to the memories. The therapist instructs the client to concentrate on the visual image, the negative belief and sensations in the body, and after that to "Let what is going to come next - happen". The client maintains this inner focus, and in the meantime moves his eyes form one side to the other, following the therapist's fingers while they move in the client's field of view (bilateral stimulation usually 20 or more sets according to the client's needs). After each set of eye movements the therapist tells the client to take a deep breath and to exhale, after which asks "What happened, what did you notice now? Hold your attention on this." Generally, the new material (image, thought, sensation or emotion) is the focus on the next set of eye movements. The standardized procedures guide the clinician-client relationship and state the continuity of the process (van den Hout \& Engelhard, 2012). This cycle of alternating attention and feedback with the clients is repeated many times during a session and it is usually accompanied by reported changes in affectivity, physiological conditions and cognitive insights (Knalfa \& Touzet, 2017). Such changes are conceptualized in the AIP model, as a result, when connections are made between the dysfunctionally stored memory and 
the more adaptive information. If these associations are not made spontaneously, the therapist intervenes, in order to give the necessary information. Because the negative images, beliefs and emotions become diffusive and less valid, the positive are inclined to become stronger and more pronounced. Bilateral stimulation is made as many times as the client shares new information or for changes that have occurred. This phase is complete, when the client states SUD rating of 0 for the original memory (Shapiro \& Maxfield, 2002).

In the fifth phase (Installation) the work is focused on connecting the desired positive cognition with the target memory or image. It can begin after the target memory is accessible without discomfort and encourages the expression and consolidation of the client's cognitive realizations. The aim is to strengthen the connections with the positive memory networks and to increase the effect of this over the ones connected with the original memory. Often, during this phase more adaptive self-perception and new positive and realistic perceptions for him appear (Korn \& Leeds, 2002; Parnell, 1996). The client simultaneously thinks about the original memory or image and the most desired positive cognition, while bilateral stimulation is being performed. The installation continues until the matter begins to acquire increasingly adaptive form. The focus is on the inclusion and increase of strength of the positive cognition, until the client assesses his confidence in the formulated positive belief at a 7, or close to 7, according the Validity of the cognition (VOC) scale.

In phase six, the one of the body scan, the goal is to finish the processing of any residual anxiety, connected to the target memory. According to the AIP model, every dysfunctionally stored information also has a physiological manifestation. EMDR processing is not considered complete, until the client is not in a condition to think for the traumatic memory without feeling any tension in his body. If the client reports any negative sensations, they are subjected to processing, until he is released of all pain, stress or discomfort (Solomon \& Shapiro, 2008).

In the seventh phase of conclusion (closure), the therapist assesses if the processing is concluded adequately or not, helps the client to be complete through the guided visualization or the self-control techniques, developed in phase two. Here the aim is to achieve the stable emotional state of the client when closing the session and to insure his emotional stability in between sessions. A short review is made through the expectations and the reorientation to the present. The attention is switched from the network, connected with the target material by ensuring redirection to other neutral or positive memory network, not connected with the target one. Clients are directed towards the need to observe and record in a diary of the material, which surfaces in between the sessions - dreams, realizations, memories, emotions. This task improves the stabilization by expanding the effect of the therapeutic process on stressful factors in real life and increasing the feeling for self-development and observation (Korn \& Leeds, 2002).

In the eighth phase (reevaluation) an assessment is made on the effects of the therapy and the provided complete processing of the therapeutic process. Besides that, the reevaluation is a part of the therapeutic plan in the beginning of every EMDR session, after the preliminary session. The therapist provides access to the memory from the previous session, studies what has appeared from the last session to the present one, in order to determine if the benefit of the adaptive processing of information is preserved. The client's diary is examined, in order to assess the extend, to which the effects of the treatment are generalized or there is a need of identifying of new paths, which must be considered. The therapist assesses if the situational influences cause distress and if the new skills for adaptive functioning are integrated in the lines of the real social system of the client. The goal of EMDR therapy is to achieve long-term beneficial effects from the process, for the shortest period of time (Shapiro \& Maxfield, 2002). The assessment of the overall process is corresponding on the processed target material, which allows the client to feel at peace with his past, to control the present and to be able to make adaptive choices for the future. The eight EMDR phases can be concluded in a few sessions or for a period of a few months, depending on the needs of the client and the severity of the symptoms. EMDR as a therapeutic approach is guided by clear and understandable principles, described in standardized procedures and protocols. Adherence to the established principles is a prerequisite for positive results of the therapy. 


\section{Case History}

Alex is a 23-year old during the time of the therapy. He's a student in a Bulgarian university but is from Kosovo. He reports a normal childhood, absence of disfunctionality in his family environment and reached stages of development, according to his own expectations. He is majoring in psychology for his bachelor degree and is managing well with the requirements of the educational program. Besides the normal stressful factors from the upcoming exams, he does not report for any other additional stressors.

The presented problem is concerned with well pronounced anxiety, caused by fear of traveling in motor vehicles, most strongly appearing during prolonged travel (around twelve hours) on a bus and/or in a car towards his home or back to Bulgaria. Although he understands the irrationality of his fears, he is not able to cope with them. The fear has manifested, earlier, in his home but has not been so intense. He does not take medication and does not approve of medication treatment.

\section{Presented Complaints}

When traveling on a bus and/or in a car from Bulgaria to his home in Kosovo, Alex is tense, worried, he cannot find composure, until he does not arrive home. He experiences the trip from his home to Bulgaria in a similar way. In the described situations, the thought that there is no restroom anywhere close or he cannot reach it is always present and this is making him feel very bad. Alex is sweating, his hands tremble, he feels discomfort in the groin, tightness in the heart, and he gets nausea, goes pale.

\section{Conceptualization of the Case}

Alex turns to a university professor, whom he trusts, for help. After familiarizing with the student's problems, the professor, who is trained in EMDR, performed a psychological-diagnostic interview and assessed the clinical status of Alex. The professor assessed that the case is suitable for EMDR and readiness for involvement in therapeutic interaction is existent. He presented the necessary information, in accordance with the principle of informed consent for the nature of EMDR and suggested Alex work with this therapeutic approach. Alex agreed to participate in EMDR therapy within the parameters of a complete therapeutic plan, developed with his active cooperation and corresponding with the desired final goal - quick achievement of relief and overcoming of the excruciating anxiety symptoms.

\section{Assessment}

A target memory was chosen for processing. Negative cognition (NG): "I am powerless" and positive cognition (PC): "I can handle that," were identified. Validity of cognition (VOC) self-reported scale of 3 , where 1 is "feels completely false," and 7 is "feels completely true," was assessed, regarding the formulated positive belief. By the Subjective Unit of Disturbance (SUD) scale was assessed, the power of the actual negative emotions, regarding the upsetting incident. A high rating of 9 was given on the SUD scale, which measures distress from 0 to 10 , with 0 representing lack of anxiety/tension and 10 representing the highest anxiety/tension.

\section{Therapeutic Process and Progress Assessment}

After the symptoms were identified and an agreement was achieved between Alex and the therapist about the problem, which would be treated, the professor started the working process with the standard three-pronged EMDR protocol (past - present - future). The essence of the EMDR approach and the ethical issues, regarding the therapy were explained. The rules of confidentiality 
for the specific positions of a client a therapist, independent from these of a student and professor, were specified. Because of the created therapeutic relationship, Alex has no problems with disclosure during the process of EMDR. A safe place was created, short and clear instructions were given about the stop signal when there is a possibility for abnormal reactions, a specific distance for the chosen eye movement was determined. The eight-phase EMDR protocol was adapted, in order to cover the needs of the specific case. The current symptomatic reactions were connected with past experiences, which were processed successfully. The current stressors that cause complaints were identified and also underwent processing. Patterns for the future were installed, by creating skills for adaptive response regarding generated situations that represent a challenge. Alex's negative belief was that he will not be able to cope with anxiety reactions, which he has, during his upcoming trip home. In the fourth phase, the desensitization, was repeated in order to resolve all channels, to reach an adaptive solution and to incorporate models of positive experience regarding the problem. Cognitive interweaving was used, in order to ease the blocked processing from Alex's past (severe anxiety to visit the restroom, far from school, when he was in primary school). The incident was seen from the perspective of the present and the thoughts of the adult: "That is ridiculous, I am 23 years old and I don't care for that anymore." In the end of this phase the SUD result dropped to 1 . In the fifth phase (installation) the therapist helped Alex to connect the desired positive cognition: "I can handle that" with the spontaneous positive experience from that point of the session. VOC was assessed as 7. In the following body scan, Alex reported that the described symptoms of discomfort and tension in his body are gone. $\mathrm{He}$ also, shared that he feels better during the session and the anxiety regarding his upcoming trip has reduced significantly, compared with the beginning of the EMDR therapy. Because the date of the trip was approaching in the phase of reevaluation, the treatment focused on assessing the effects of the therapy, more precisely are present the low anxiety levels on SUDS, are high VOC indicators maintained and do the physical disturbances are still absent. It was examined if a new material for work has manifested between the sessions as a reference for future work and formulation of new therapeutic goals. A total of nine EMDR sessions were conducted with Alex before the trip. In the beginning of the EMDR process the validity of positive cognition was assessed as 3, in the phase of installation it was increased to a 7. The process of desensitization continued until Alex's initial SUDS score dropped from 9 to 1 .

\section{Clinical Issues and Summary}

In Alex's case the impact of EMDR on fear from traveling by bus or by car is researched. It follows how the processing of information, activated during EMDR, leads to a significant decrease of anxiety symptoms. The relatively fast positive result of the therapeutic process suggests that EMDR could be effectively applied to cope with the fear from traveling with motor vehicles. It can be supposed that the result is affected by Alex's motivation to overcome the problem with therapy (without medications), as well as the need to travel with comfort to his relatives and back. The resources, which he possesses - adequate social incorporation, skills for appropriate self- revelation, handling the educational process were some of the factors that supported the good prediction of the therapy. In the email send by Alex after the trip home, the low levels of anxiety, in comparison with all previous trips (before EMDR), are confirmed. Alex, also, states willingness to continue the therapy after his return, in order to achieve complete elimination of the slight anxiety attacks, which we can explain with incompleteness of the process (SUD 1) because of the upcoming trip. Although, EMDR seems like an effective method for overcoming the fear of travelling with motor vehicles, conducting a controlled study about the possibilities of the approach on this issue would be significant for the therapeutic practice.

\section{References}

De Jongh, A., Ten Broeke, E., \& Meije, S. (2010). Two method approach: A case conceptualization model in the context of EMDR. Journal of EMDR Practice and Research, 4 (1), 12-21. 
Engelhard, I. M., van Uijen, S., \& ven den Hout, M. A. (2010). The impact of taxing working memory on negaVol. 12, No. 1, 2018 tive and positive memories. European Journal of Psychotraumatology, 1, 23-29.

Jeffries, F. W., \& Davis, P. (2012). What is the role of eye movements in eye movement desensitization and reprocessing (EMDR) for post-traumatic stress disorder (PTSD)? A review. Behavioural and Cognitive Psychotherapy, 41 (3), 290-300. doi:10.1017/S1352465812000793.

Korn, D. L., \& Leeds, A. M. (2002). Preliminary evidence of efficacy for EMDR resource development and installation in the stabilization phase of treatment of complex posttraumatic stress disorder. Journal of Clinical Psychology, 58 (12), 1465-1487.

Knalfa, S., \& Touzet, CF. (2017). EMDR therapy mechanisms explained by the theory of neural cognition. Journal of Trauma Stress Disorders \& Treatment, 6 (4), doi: 10.4172/2324-8947. 1000179.

McCullough, L. (2002). Exploring change mechanisms in EMDR applied to "small t trauma" in short term dynamic psychotherapy: research questions and speculations. Journal of Clinical Psychology, 58, 1465-1487.

Maxfield, L., Melnyk, W. T., \& Gordon Hayman, C. A. (2008). A working memory explanation for the effects of eye movements in EMDR. Journal of EMDR Practice and Research, 2 (4), 247-261.

Parnell, L. (1996). Eye movement desensitization and reprocessing (EMDR) and spiritual unfolding. The Journal of Transpersonal Psychology, 28 (2), 129 -153.

Patihis, L., Cruz, C. S., \& McNally, R. J. (in press). Eye movement desensitization and reprocessing (EMDR). In V. Zeigler-Hill \& T. K. Shackelford (Eds.) Encyclopedia of personality \& individual differences. New York, NY: Springer. doi: 10.1007/978-3-319-28099-8_895-1.

Rogers, S., \& Silver, S. (2002). Is EMDR an exposure therapy? A review of trauma protocols. Journal of Clinical Psychology, 58 (1), 43-59.

Shapiro, F. (2014). The role of eye movement desensitization and reprocessing (EMDR) therapy in medicine: Addressing the psychological and physical symptoms stemming from adverse life experiences. The Permanente Journal, 18 (1), 71-77.

Shapiro, F., \& Maxfield, L. (2002). Eye movement desensitization and reprocessing (EMDR): Information processing in the treatment of trauma. Journal of Clinical Psychology/In Session: Psychotherapy in Practice, 58 (8), 933-946.

Solomon, R. M., \& Shapiro, F. (2008). EMDR and the adaptive information processing model: Potential mechanisms of change. Journal of EMDR Practice and Research, 2 (4), 315-325.

Valiente-Gómez, A., Moreno-Alcázar, A., Treen, D., Cedrón, C., Colom, F., Pérez, V., \& Amann, B. L. (2017). EMDR beyond PTSD: A systematic literature review. Frontiers in Psychology, 8, 1668. doi: 10.3389/ fpsyg.2017.01668.

van der Vleugel, B., van den Berg, D. P. G., \& Staring, A. T. (2012). Trauma, psychosis, post-traumatic stress disorder and the application of EMDR. Supplemento alla Rivista di psichiatria, 47 (2), 33-38.

van den Hout, M., \& Engelhard, I. (2012). How does EMDR work? Journal of Experimental Psychopathology, $3(5), 724-738$.

Received: May 20, 2018

Accepted: June 26, 2018 